\title{
THE EQUIVALENCE OF HIGH SUBGROUPS
}

\author{
PAUL HILL ${ }^{1}$
}

\begin{abstract}
Two subgroups of a group $G$ are called equivalent if there is an automorphism of $G$ that maps one of the subgroups onto the other. Suppose that $G$ is a $p$-primary abelian group and that $\lambda$ is an ordinal. A subgroup $H$ of $G$ is $p^{\lambda}$-high in $G$ if $H$ is maximal in $G$ with respect to having zero intersection with $p^{\lambda} G$. Under certain conditions on the quotient group $G / p^{\lambda} G$ slightly weaker than total projectivity, it is shown, for a given $\lambda$, that any two $p^{\lambda}$-high subgroups of $G$ are equivalent. In particular, if $G / p^{\omega} G$ is $p^{\omega+1}$-projective, the $p^{\omega}$-high subgroups of $G$ are all equivalent.
\end{abstract}

D. Cutler [1] has recently proved the following result, where $f_{\alpha}(G)$ denotes the $\alpha$ th Ulm invariant of $G$.

THEOREM. Let $G$ be an abelian p-group such that $G / p^{\omega} G$ is $p^{\omega+n}$-projective, and if $n>1$ then $f_{\omega+m}(G)=0$ for $0 \leqslant m<n-1$. Then any two high subgroups of $G$ are isomorphic.

Recall that $H$ is called a high subgroup of the abelian $p$-group $G$ if $H$ is maximal with respect to having zero intersection with $p^{\omega} G$. More generally, if $H$ is maximal with respect to having zero intersection with $p^{\lambda} G$, then $H$ is said to be $p^{\lambda}$-high in $G$. Notation and terminology generally are in agreement with [2].

The case $n=1$ in Cutler's theorem avoids certain technicalities, gains simplicity, and is of special interest.

COROLlary. Let $G$ be an abelian p-group such that $G / p^{\omega} G$ is $p^{\omega+1}$-projective. Then any two high subgroups of $G$ are isomorphic.

If $G / p^{\omega} G$ satisfies the stronger hypothesis of being $p^{\omega}$-projective, which is equivalent to being a direct sum of cyclic groups, the preceding corollary is contained in the pioneer work on high subgroups by J. Irwin and E. Walker [7], who first raised and developed interest in the question: for which abelian $p$-groups $G$ are all of its high subgroups isomorphic? As Cutler mentioned in his paper, the first example of a group $G$ with nonisomorphic high subgroups was constructed in [3]. However, it was soon apparent through the joint work of the author and C. Megibben [6] that the case of possessing nonisomorphic high subgroups is the rule rather than the

Received by the editors August 1, 1982.

1980 Mathematics Subject Classification. Primary 20K10, 20K27.

Key words and phrases. Abelian $p$-groups, high subgroup, equivalent subgroups, $p^{\alpha}$-projective, extending isomorphisms, automorphism.

'Sponsored by NSF Grant MCS 8102470. 
exception. Thus the positive became accentuated, and interest was added to results in the direction of those of Cutler stated above.

In this paper we are able to improve Cutler's theorem in two ways. First, we generalize from the special ordinal $\omega$ to an arbitrary ordinal $\lambda$, but this requires replacing " $G / p^{\lambda} G$ is $p^{\lambda+n}$-projective" by "there is a nice, $p^{n}$-bounded subgroup $B / p^{\lambda} G$ of $G / p^{\lambda} G$ where $G / B$ is totally projective." The two statements are equivalent for $\lambda=\omega$; in fact, the latter condition is the one actually used by Cutler in his proof. Nevertheless, to be precise we should say that we generalize the theorem logically equivalent to Cutler's obtained from it by replacing the first quotation above by the latter. Secondly, we strengthen even the generalized theorem by proving that two high subgroups $H$ and $K$ are more than isomorphic. They are equivalent! By this we mean that there is an automorphism of $G$ that maps $H$ onto $K$. Actually, a little more is true. What we prove is the following.

THEOREM. Let $H$ and $K$ be $p^{\lambda}$-high subgroups of the p-primary group $G$. Let $n$ denote a nonnegative integer and suppose that the $(\lambda+m)-U l m$ invariant of $G$ is zero whenever $0 \leqslant m<n-1$; the condition is satisfied vacuously if $n \leqslant 1$. Provided that $G / B$ is totally projective for a $p^{n}$-bounded and nice subgroup $B / p^{\lambda} G$ of $G / p^{\lambda} G$, there exists an automorphism of $G$ that leaves the elements of $p^{\lambda} G$ fixed and maps $H$ onto $K$.

Before we begin the formal proof of the theorem, we make some observations and prove an essential lemma. The vanishing of the initial Ulm invariants of $p^{\lambda} G$ implies

$$
p^{\lambda} G\left[p^{m}\right] \subseteq p^{n-m}\left(p^{\lambda} G\right) \text { if } m \leqslant n,
$$

and

$$
G\left[p^{n}\right]=H\left[p^{n}\right] \oplus p^{\lambda} G\left[p^{n}\right]=K\left[p^{n}\right] \oplus p^{\lambda} G\left[p^{n}\right] .
$$

Let $\eta: H\left[p^{n}\right] \nrightarrow K\left[p^{n}\right]$ denote the natural isomorphism associated with (2); $\eta(x)=y$ if $x \in H\left[p^{n}\right], y \in K\left[p^{n}\right], z \in p^{\lambda} G$, and if $x=y+z$. Observe that if $p^{n}\left(B / p^{\lambda} G\right)=0$, in accordance with the hypothesis of the theorem, then $H \cap B$ and $K \cap B$ are contained in $H\left[p^{n}\right]$ and $K\left[p^{n}\right.$ ], respectively.

LEMMA. With the notation and hypothesis of the theorem, there exists an automorphism $\pi$ of $B$ that satisfies the following conditions:

(a) $\pi$ preserves heights computed in $G$.

(b) $\pi$ is the identity on $p^{\lambda} G$.

(c) $\pi$ agrees with $\eta$ on $B \cap H$.

Proof. Set $B_{0}=(B \cap H) \oplus p^{\lambda} G=(B \cap K) \oplus p^{\lambda} G$. Since $B \supseteq p^{\lambda} G$, the isomorphism $\eta$ maps $B \cap H$ onto $B \cap K$. Let $\pi_{0}$ be the automorphism of $B_{0}$ that agrees with $\eta$ on $B \cap H$ and is the identity on $p^{\lambda} G$. Note that $\pi_{0}$ preserves heights in $G$; in fact, $\pi_{0}(x)-x \in p^{\lambda} G$ if $x \in B_{0}$ and $\pi_{0}(x)-x=0$ if $x \in p^{\lambda} G$. Thus $\pi_{0}$ satisfies conditions (a)-(c). In order to demonstrate that $\pi_{0}$ can be extended not only to an automorphism $\pi$ of $B$ but to one that continues to preserve heights in $G$, we write the bounded $p$-group $B / B_{0}$ as a direct sum of cyclic groups. Let

$$
B / B_{0}=\sum_{i \in I}\left\langle b_{i}+B_{0}\right\rangle
$$


where $o\left(b_{i}+B_{0}\right)=p^{m_{i}}$. We know that $m_{i} \leqslant n$ for each $i$. Since $B_{0}=(B \cap H) \oplus$ $p^{\lambda} G$, we can write $p^{m_{i}} b_{i}=h_{i}+w_{i}$, where $h_{i} \in B \cap H$ and $w_{i} \in p^{\lambda} G$. Since $p^{n-m_{i}} h_{i}$ $=0$, we know that $h_{i} \in K\left[p^{n-m_{i}}\right] \oplus p^{\lambda} G\left[p^{n-m_{i}}\right]$. Thus $h_{i}=y_{i}+z_{i}$, where $y_{i} \in B$ $\cap K$ and $z_{i} \in p^{\lambda} G\left[p^{n-m_{i}}\right] \subseteq p^{m_{i}}\left(p^{\lambda} G\right)$ according to (1). For each $i$, choose $u_{i} \in p^{\lambda} G$ so that $p^{m_{i}} u_{i}=z_{i}$. Observe that $\pi_{0}\left(h_{i}\right)=y_{i}=h_{i}-p^{m_{i}} u_{i}$. Consider an extension $\pi$ : $B \rightarrow B$ of $\pi_{0}: B_{0} \nrightarrow B_{0}$ defined by $\pi\left(b_{i}\right)=b_{i}-u_{i}$; more generally, $\pi$ is defined by

$$
\pi\left(\left(\sum t_{i} b_{i}\right)+a\right)=\left(\sum t_{i}\left(b_{i}-u_{i}\right)\right)+\pi_{0}(a)
$$

if $t_{i}$ is an integer and $a \in B_{0}$. Since

$$
\pi\left(p^{m_{i}} b_{i}\right)=p^{m_{i}} b_{i}-z_{i}=\left(h_{i}+w_{i}\right)-z_{i}=\pi_{0}\left(p^{m_{i}} b_{i}\right)
$$

shows that $\pi$ agrees with $\pi_{0}$ on $\left\langle b_{i}\right\rangle \cap B_{0}$, and since the generators $\left\{b_{i}\right\}_{i \in I}$ are independent $\bmod B_{0}$, it is routine to verify that $\pi$ is well defined. Since $\pi$ extends $\pi_{0}$, and since $\pi(x)-x \in p^{\lambda} G$ for all $x \in B$, it is immediate that $\pi$ preserves heights in $G$ and is, in fact, an automorphism of $B$ that satisfies conditions (a)-(c). Thus the Lemma is proved.

Proof of Theorem. According to the Lemma, there is a height-preserving automorphism $\pi$ of $B$ that leaves $p^{\lambda} G$ fixed and maps $B \cap H$ onto $B \cap K$. We intend to extend $\pi$ to an automorphism of $G$ that maps $H$ onto $K$.

Since $B$ is nice in $G$ and since $G / B$ is totally projective, there exists a collection $\bigodot$ of nice subgroups of $G$, one of which is equal to $B$ and each of which contains $B$, with the property that if $N \in \mathcal{C}$ and $A$ is any countable subgroup of $G$, there exists $N^{\prime} \in \mathcal{C}$ containing both $N$ and $A$ with $N^{\prime} / N$ countable. Indeed, there exists such a collection $\mathcal{C}$ that is closed with respect to group union because $G / B$ satisfies the third axiom of countability.

Suppose that $\pi: M \gg M^{\prime}$ is an isomorphism that preserves heights in $G$, where (i) $M$ and $M^{\prime}$ are both finite extensions in $G$ of some fixed $N \in \mathcal{C}$ for which the restriction of $\pi$ is an automorphism, and (ii) $\pi(M \cap H)=M^{\prime} \cap K$. The above is indeed the case for $M=B=M^{\prime}$. It is enough to show that the preceding conditions on $\pi$ can all be retained for some extension of $\pi$ to $\langle M, x\rangle$ whenever $x \in G$. Due to the symmetry of $M$ and $M^{\prime}$, including the reversibility of the isomorphism $\pi$ and the fact that (i) ensures that $M=G$ if and only if $M^{\prime}=G$, such local extensions of $\pi$ repeated a countable number of times would enable us to reach simultaneously with domain and range an $N^{\prime} \in \mathcal{C}$ for which $N^{\prime} \supseteq M \cup M^{\prime} \supseteq N$. Then the whole process repeats (or is finished if $N$ is chosen maximal by an application of Zorn's lemma). Before we complete the proof of the theorem by showing that the desired local extension of $\pi$ exists, one relevant, but side, remark is made. If it were not for condition (ii) it would follow at once from known results that the local extension is possible. In fact, the case where (ii) is deleted goes back to [4]. However, condition (ii) is the whole point of the present theorem. When we are finished, we want $\pi$ to map $H$ onto $K$. But even for the case at hand where (ii) is to be honored, there is a proof in the literature of a similar result for countable groups that will serve as a model for the present proof. Reference is made to the proof of Theorem 2 in [5]. We simply make the necessary and appropriate modifications and adjustments. The outline is the same. 
Let $x \in G$. In order to show that $\pi$ extends to a height-preserving isomorphism from $\langle M, x\rangle$ into $G$ such that $\pi(\langle M, x\rangle \cap H)=\left\langle M^{\prime}, \pi(x)\right\rangle \cap K$, we may assume without loss of generality that $p x \in M$ but $x \notin M$. As usual, we choose $x$ (in the coset $x+M$ ) proper with respect to $M$. Let $h(x)$ denote the height of $x$; all heights are computed in $G$. For simplicity of notation, set $h(x)=\alpha<\lambda$. It is essential that $x$ be proper with respect to $M$, but after that consideration, preference is given to $h(p x)>\alpha+1$. Thus if there exists $m \in M \cap p^{\alpha} G$ such that $h(p(x+m))>\alpha+1$, and if $h(p x)=\alpha+1$, the latter does not stand, but $x$ is replaced by $x+m$. In other words, we choose $x$ so that $h(p x)>\alpha+1$ if such a choice is possible among the proper elements of the coset $x+M$. Since this choice is not always available, there are two cases: $h(p x)=\alpha+1$ and $h(p x)>\alpha+1$. Each of these cases has three subcases: $x \in H, x \notin H$ but $x \in\langle H, M\rangle$, and $x \notin\langle H, M\rangle$. In each of six (sub)cases, we seek a suitable element $y$ upon which to map $x$.

Case 1.1. $h(p x)=\alpha+1, x \in H$. Since $\pi(p x) \in p^{\alpha+1} G \cap K=p^{\alpha+1} K$, we choose $y \in K \cap p^{\alpha} G=p^{\alpha} K$ such that $p y=\pi(p x)$.

Case 1.2. $h(p x)=\alpha+1, x \notin H$ but $x+m \in H$ for some $m \in M$. Choose $y_{0} \in p^{\alpha} G$ so that $p y_{0}=\pi(p x)$. If $y_{0}+\pi(m) \in K$, set $y=y_{0}$. If $y_{0}+\pi(m) \notin K$, observe that $p\left(y_{0}+\pi(m)\right)=\pi(p(x+m)) \in p K$ and, therefore, $p\left(y_{0}+\pi(m)\right)=p k$ for some $k \in K$. Consequently, $y_{0}+\pi(m)=k+k_{0}+z_{0}$, where $k_{0} \in K[p]$ and $z_{0} \in p^{\lambda} G[p]$ since $G[p]=K[p] \oplus p^{\lambda} G[p]$. Set $y=y_{0}-z_{0}$ and observe that $y+$ $\pi(m) \in K$.

Case 1.3. $h(p x)=\alpha+1, x \notin\langle H, M\rangle$. Choose $y \in p^{\alpha} G$ so that $p y=\pi(p x)$. It is easy to verify that $y \notin\left\langle K, M^{\prime}\right\rangle$.

Case 2.1. $h(p x)>\alpha+1, x \in H$. In this case, $p x \in p^{\alpha+2} H$. Therefore, $p x=p h$ where $h \in p^{\alpha+1} H$ and $p(x-h)=0$. Moreover, the element $x-h$ has height exactly $\alpha$ and is proper with respect to $M$. Likewise, there must exist an element $s \in G[p]$ with height exactly $\alpha$ and proper with respect to $M^{\prime}$, for $\pi$ preserves heights in $G$ and there exists $N \in \mathcal{C}$ such that the following diagram is commutative, where $M$ and $M^{\prime}$ are finite extensions of $N$ :

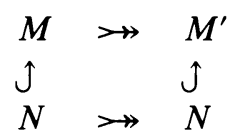

The above implies that the Ulm invariants of $G$ relative to $M$ are the same as those of $G$ relative to $M^{\prime}$. Since $s \in G[p]=K[p] \oplus p^{\lambda} G[p]$, and since $\alpha<\lambda$, we may assume without loss of generality that $s \in K$. Choose $w \in p^{\alpha+1} K$, so that $p w=$ $\pi(p x)$, and set $y=w+s \in K$.

Case 2.2. $h(p x)>\alpha+1, x \notin H$ but $x+m \in H$ for some $m \in M$. Choose $w \in p^{\alpha+1} G$ so that $p w=\pi(p x)$. By an argument similar to that of the preceding case, there exists $s \in G[p]$ that is proper with respect to $M^{\prime}$ and has height exactly $\alpha$. Set $y_{0}=w+s$. If $y_{0}+\pi(m) \in K$, let $y=y_{0}$. If $y_{0}+\pi(m) \notin K$, define $y$ in terms of $y_{0}$ exactly as in Case 1.2 and obtain $y+\pi(m) \in K$.

Case 2.3. $h(p x)>\alpha+1, x \notin\langle H, M\rangle$. Choose $w \in p^{\alpha+1} G$ so that $p w=\pi(p x)$ and choose $s \in G[p]$ that is proper with respect to $M^{\prime}$ and has height exactly $\alpha$. Set $y=w+s$. Observe that $y \notin\left\langle K, M^{\prime}\right\rangle$. 
In all six cases, we have chosen $y$ so that $x \rightarrow y$ extends $\pi$ to an isomorphism $\langle M, x\rangle \gg\left\langle M^{\prime}, y\right\rangle$ that continues to preserve heights in $G$, and $\pi(\langle M, x\rangle \cap H)=$ $\left\langle M^{\prime}, y\right\rangle \cap K$. This completes the proof of the theorem.

Corollary 1. Let $G$ be an abelian p-group such that $G / p^{\omega} G$ is $p^{\omega+1}$-projective. Then any two high subgroup of $G$ are equivalent.

COROllary 2. Let $G$ be an abelian p-group and let $\lambda$ denote an ordinal. If $G / B$ is totally projective, where $B \supseteq p^{\lambda} G \supseteq p B$ and $B$ is nice in $G$. then any two $p^{\lambda}$-high subgroups of $G$ are equivalent.

COROllaRY 3. Any two $p^{\lambda}$-high subgroups of a totally projective group are equivalent.

A solution to the following problem may provide some additional insight into the long-standing question: when are all the high subgroups of a $p$-group $G$ isomorphic?

Problem. Find an example, if possible, of an abelian $p$-group $A$ of length $\lambda$ such that all $p^{\lambda}$-high subgroups of $G$ are isomorphic whenever $G / p^{\lambda} G=A$, but not equivalent.

\section{REFERENCES}

1. D. Cutler, Primary abelian groups having all high subgroups isomorphic, Proc. Amer. Math. Soc. 83 (1981), 467-470).

2. L. Fuchs, Infinite ahelian groups, Vols. 1, 2. Academic Press, New York, 1970, 1973.

3. P. Hill, Certain pure subgroups of primary groups, Topics in Abelian Groups, Scott, Foresman, Chicago, Ill., 1963.

4. On the classification of ahelian groups, photocopied manuscript, Houston, 1967.

5. Automorphisms of countable primary abelian groups, Proc. Amer. Math. Soc. 25 (1971), 135-140.

6. P. Hill and C. Megibben, On primary groups with countable basic subgroups, Trans. Amer. Math. Soc. 124 (1966), 49-59.

7. J. Irwin and E. Walker, On N-high subgroups of abelian groups, Pacific J. Math. 11 (1961), 1363-1374.

Department of Mathematics, Auburn University, Auburn University, Alabama, 36849 